\title{
Cystic Tumours of the Pancreas: A Challenging Pathology, Diagnosis and Management
}

\author{
Abd Elrafea Elkak \\ The Royal Hospital, Muscat, Oman \\ Email: aeraelkak@yahoo.co.uk
}

How to cite this paper: Elkak, A.E. (2016) Cystic Tumours of the Pancreas: A Challenging Pathology, Diagnosis and Management. Journal of Cancer Therapy, 7, 712728.

http://dx.doi.org/10.4236/jct.2016.710073

Received: September 1, 2016

Accepted: September 25, 2016

Published: September 28, 2016

Copyright $\odot 2016$ by author and Scientific Research Publishing Inc. This work is licensed under the Creative Commons Attribution International License (CC BY 4.0).

http://creativecommons.org/licenses/by/4.0/ c) (i) Open Access

\begin{abstract}
Background: Cystic tumours of the pancreas are relatively uncommon tumours but there is an increasing awareness of their importance. Modern advances in imaging had resulted in a more prompt diagnosis of these tumours. The understanding of the pathology, clinical features, diagnosis and management of these tumours is continuously evolving. Data Sources: Systematic literature review. A PubMed database research was performed. Relevant articles published in English were identified and scrutinized. Duplications of information and persistently unsolved uncertainties were excluded. Results: Cystic tumours of the pancreas should be differentiated from pancreatic pseudocysts. Cystic tumours could be classified into: cystic serous neoplasms (SCN), mucinous cystic neoplasms ( $\mathrm{MCN})$, intraductal papillary neoplasms (IPMN), solid pseudopapillary neoplasms (SPPN) and the rarer tumours cystic pancreatic endocrine neoplasms (PEN). Except for SCN, all these tumours should be regarded, at least, as potentially malignant but they may be frankly malignant. The age of the patient and site of the lesion may be helpful in the diagnosis. In particular, MCN is always encountered in females in their middle age and often in the body and tail of the pancreas. Many of these tumours are diagnosed incidentally. Pancreatitis and hyperamylasaemia should be interpretted with caution as IPMN may present as pancreatitis. CT is the primary diagnostic tool although MRI, EUS, PET, abdominal ultrasound and ERCP have all been utilised. Biochemical markers are of limited value in the diagnosis. Conclusions: Management decision depends primarily on the understanding of pathology and on how confident the preoperative diagnosis is. SCN may be observed. Partial pancreatectomy is the usual operation performed for most other tumours according to the location of the lesion which means that distal pancreatectomy is usually the operation performed for MCN. Total pancreatectomy for IPMN, enucleation and central pancreatectomy have all been described in the literature. Laparoscopy is more suitable for benign or low grade malignant tumours in the context of distal pancreatectomy. Spleen should be preserved unless splenectomy is
\end{abstract}


indicated and splenic vessels should be preserved wherever possible when preserving the spleen.

\section{Keywords}

Pancreatic Cystic Tumours, IPMN, Laparoscopic Pancreatic Surgery

\section{Introduction}

Cystic tumours of the pancreas have been increasingly recognized in the last two decades [1]. Generally speaking, most of these tumours are either serous or mucinous neoplasms [2] [3]. Their malignant potential has to be well emphasized [1] [2]. Because of the aging of the population, prevalence of medical checkups, and advances in imaging studies, the number of incidentally detected pancreatic cystic lesions has increased [4]. The approach to incidentally noted pancreatic cysts is constantly evolving [5]. Parameters including symptoms, cyst size, presence of solid components, radiological appearance and pancreatic duct involvement have all to be taken into consideration when deciding which patients would benefit from surgical resection [5]. Neoplastic cysts should be differentiated from non-neoplastic cysts and further differentiation between benign and malignant cysts should be made [4]. The surgeon has to have good understanding of these tumours to minimize the chance of missing what could turn to be a malignant tumour. This review aims to cover the pathology, diagnosis and different management options of these tumours with some emphasis on the role of laparoscopic surgery.

\section{Epidemiology and Prevalence}

Cystic neoplasms of the pancreas are relatively uncommon conditions of the pancreas comprising less than $1 \%$ of primary pancreatic tumours but are becoming increasingly important in clinical practice because of the advancement of the diagnostic modalities used to detect asymptomatic lesions [6], and therefore, they are much more common than previously appreciated. Between $10 \%$ and $15 \%$ of pancreatic cystic lesions are primary cystic neoplasms whilst most of the remaining majority are pseudocysts [7]-[9].

\section{Pathology}

\subsection{Classification}

Cystic tumours of the pancreas can be classified into [3] [7] [10]:

1) Cystic serous neoplasms (SCN): Previously known as serous cystadenoma. Serous cystadenocarcinoma is very rare.

2) Mucinous cystic neoplasms (MCN): which could be further classified into mucinous cystadenoma, mucinous cystadenoma with mild dysplasia, mucinous cystadenocarcinoma non-infiltrating and mucinous cystadenocarcinoma infiltrating.

3) Intraductal papillary neoplasms (IPMN) which are further categorised into intraductal papillary mucinous adenoma, IPMN with mild dysplasia, intraductal papillary 
mucinous cystadenocarcinoma non-infiltrating and intraductal papillary mucinous cystadenocarcinoma infiltrating.

4) Solid pseudopapillary neoplasms (SPPN).

5) Rarer tumours: cystic pancreatic endocrine neoplasms (PEN), cystic ductal adenocarcinoma and acinar cell cystadenoma.

Mucin producing cystic tumours of the pancreas would, therefore, comprise MCN and IPMN.

\subsection{Description of the Main Pathological Features}

Cystic serous neoplasms (SCN): Represent 25\% - 30\% of primary cystic tumours of the pancreas [8]. These tumours are more common females [8] with the mean age being 62 years [10] and are evenly distributed throughout the pancreas [10]. They exhibit macroscopic variation and can be divided into two categories: serous microcystic and serous oligocystic [3] [8].

Mucinous cystic neoplasms (MCN): Represent $40 \%-50 \%$ of primary cystic tumours of the pancreas [7]. These tumours are formed of mucin producing cells and are characterised by the presence of ovarian like stoma [10] which stains for human chorionic gonadotropin in $62 \%$ of cases [11] although non ovarian stoma MCNs have been reported [7]. They always occur in females in their middle age i.e. in the 40 to 50 year age range [12] [13]. They are often found in the body and tail of the pancreas [10] [12].

Intraductal papillary neoplasms (IPMN): These tumours, reported first in 1982 by Ohhashi et al., make up 20\% - 30\% of primary cystic tumours of the pancreas [14]-[16]. They are found most often in male patients in their $60 \mathrm{~s}$ and $70 \mathrm{~s}$ although they have been reported in females [7] [11]. They can occur anywhere in the pancreas [6] but are more often in the pancreatic head and neck region [7] i.e. they are more common in, but not confined to, the head and neck of pancreas. They have been reported to arise in association with Peutz-Jeghers syndrome and familial adenomatous polyposis [11]. These tumours produce mucin like MCN but they are a different category [6]. They always communicate with the pancreatic duct, a feature not seen in MCN [7]. The lesions can be divided into two categories: 1) Main duct type and 2) Branch duct type [10]. Unlike MCN, SCN and IPMN lack ovarian-type stroma [17]. The potential for multifocality and late recurrence has made surgical management of IPMN quite challenging [17].

Solid pseudopapillary neoplasms (SPPN): Are a rare clinical entity [18]. SPPN were described by Frantz in 1959 [18]. Different synonyms have been used (solid and cystic papillary neoplasms, papillary cystic neoplasms, Frantz's tumours, papillary and cystic epithelial neoplasms) [18]. These tumours occur more often in young women mostly in the second and third decades of life although have been reported in men and are evenly distributed throughout the pancreas [7] [18]-[20]. They are usually large well encapsulated tumours sharply demarcated from the pancreatic tissues and have a mixture of solid and cystic components with a papillary pattern [18] [19]. They can be misdiagnosed as acinar cell carcinoma, non-functioning islet cell tumour, cystadenoma or 
even adenocarcinoma [17]. Due to the paucity of these tumours, the natural history of the disease is not fully understood [19].

Cystic pancreatic endocrine neoplasms (PEN): They are cystic variants of their solid counterparts which can give a picture similar to the cystic tumours of the pancreas described above [6].

\subsection{Risk of Malignancy}

Broadly speaking these tumours are classified into benign, potentially malignant and malignant [10].

Cystic serous neoplasms (SCN): are generally considered benign. Malignant changes in SCN are extremely rare [3] [21]. Indeed, the interpretation of these cases as malignant has been controversial [8].

Mucinous cystic neoplasms ( $\mathrm{MCN})$ : are at least potentially malignant [10] with a malignant potential ranging from $6 \%$ to $36 \%$ [7]. The prognosis after resection is better than ductal adenocarcinoma of the pancreas [6].

Intraductal papillary neoplasms (IPMN): Main duct tumours have a higher chance of malignancy, which could be invasive or non invasive carcinoma compared with branch duct tumours (between $60 \%$ and $90 \%$ for the former and $6 \%$ and $46 \%$ for the latter respectively) [11] [14] [15]. IPMNs may range from premalignant lesions with low-grade dysplasia to invasive malignancy and they have a clear tendency to become invasive carcinoma [13]. As with mucinous cystadenocarcinoma, invasive IPMN is reported to recur after margin negative pancreatic resection [17] but unlike benign $M C N$, noninvasive IPMNs have been reported to recur after R0 pancreatic resections [17].

Prognosis of a ductal carcinoma developing from IPMN does not differ significantly from "classic" ductal adenocarcinoma, with a very poor (10\%) 5-year survival rate [16]. However, prognosis of IPMN can still be regarded favourable, because the survival rate can be as high as $70 \%$ if the tumour is non-invasive [16].

Solid pseudopapillary neoplasms (SPPN): They are generally regarded benign or tumours with low grade malignant potential but have been reported to grow to a large size, infiltrate surrounding organs and vessels, metastasise to the liver and in the peritoneal cavity to produce Krukenburg-type distant metastasis and indeed recur after radical surgical excision [10] [18] [19] but they can run a very indolent course [10]. Prognosis is excellent after resection [18].

The behaviour of the cystic pancreatic endocrine neoplasms (PEN) is similar to their solid counterparts [6].

\section{Diagnosis}

$40 \%-75 \%$ of these lesions are asymptomatic diagnosed incidentally in the course of investigating other abdominal conditions [9]. There are two important questions to answer. The first question is how to differentiate a cystic pancreatic tumours from apancreatic pseudocyst. The other question is the nature of the lesion and whether it is benign or malignant. Taking careful clinical history and using the imaging modalities 
and biochemical markers described below could be helpful to establish a preoperative diagnosis although making a definitive preoperative diagnosis may still not be possible [22]. Caution should be exercised when interpreting the clinical and radiological findings. In particular, clinical history of pancreatitis and hyperamylasaemia should be interpreted with caution as IPMN can present with acute pancreatitis by compression of the pancreatic duct or occlusion of the pancreatic secretion [22] [23]. Indeed, mucinous cystadenoma with histologically proved ovarian like stroma has been reported to present with acute pancreatitis [24]. Moreover, communication with pancreatic duct on MRI may also be a misleading sign as it is reported to happen both in pseudocysts and IPMN [8] [23]. Inadequate management or even inappropriate surgical treatment is not unknown if a cystic tumour is mistaken for a pseudocyst [16].

Historically, $80 \%$ - 90\% of cystic lesions were considered to be pseudocysts lacking an epithelial lining on the inner cyst surface and $10 \%-20 \%$ are true cysts with an epithelial lining on the inner cyst surface [8] [23]. Many of these true cysts are now known to be cystic tumours [24]. Furthermore, although pseudocysts always lack epithelial lining, the lack of epithelial lining does not rule out cystic tumours [25].

The diagnostic tools are primarily imaging and biochemical studies.

\subsection{Imaging}

CT scan: CT with intravenous contrast scan is often employed as the main diagnostic modality [7]

SCN: The pathognomoic feature of the microcystic SCN is the "honeycomb" appearance [9] where many small cysts are found within the larger cyst [10]. A central stellate scar is often seen in the centre of the SCA and is considered pathognomonic [7]. Sunburst pattern of calcification is also diagnostic [8] [9]. The oligocystic adenoma appears macrocystic rather than microcystic and can be misdiagnosed as MCN or pseudocysts [8] (Figure 1(a) and (b)).

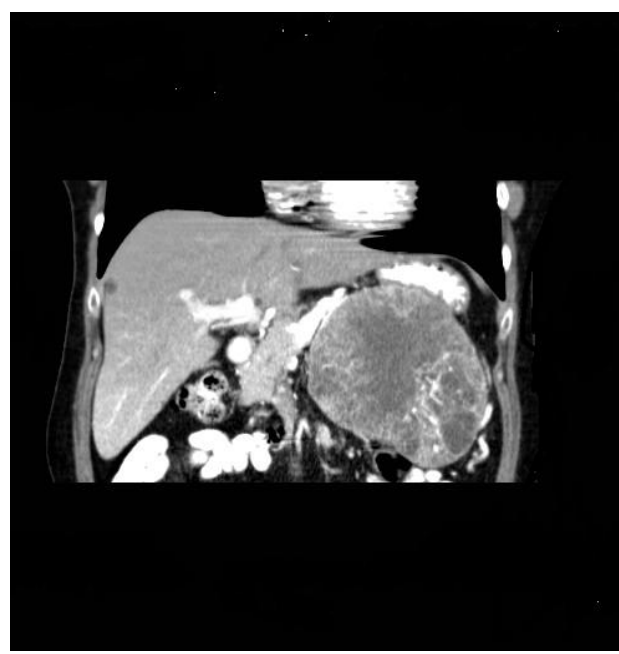

(a)

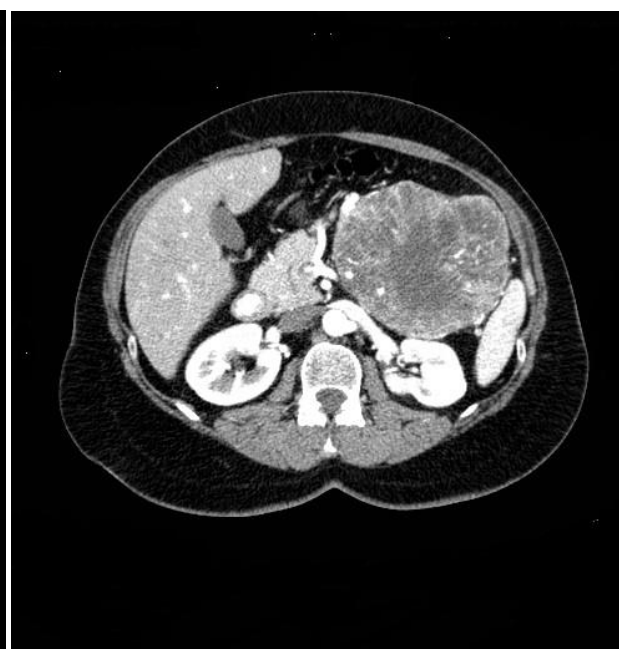

(b)

Figure 1. Microcystic serous cystadenoma. 
MCN: MCN usually appear unilocular but could be multilocular [6]. MCN are often macrocytic although microcystic lesions occur and are characteristically multilocular [7]. Dilatation of pancreatic duct is uncommon and pancreatic duct communication is rare in SCN and MCN [7]. Features of malignancy are size more than $2-3 \mathrm{~cm}$, septal enhancement, wall nodularity, calcification, solid component within the cyst and local infiltration and distant metastasis [6].

IPMN: Main duct IPMN appears as a segmentally dilated tortuous pancreatic duct with filling defects [6]. Brach duct IPMN appears as a unilocular cyst [6]. Nodules in the main duct system and dilatation of the main duct $(>1 \mathrm{~cm})$ suggest main duct IPMN whilst a mucinous cyst communicating with the pancreatic duct outside a main duct of normal diameter indicates branch duct IPMN [11]. Communication with the pancreatic duct helps distinguish branch duct IPMN from other cystic lesions of the pancreas [8]. Multiplicity of the cysts, multifocality of the lesion and pancreatic ductal dilatation distal to the lesion favour the diagnosis of IPMN. The presence of a mural nodule often signifies an invasive cystic neoplasm [7].

SPPN: SPPN is a well capsulated large lesion with a heterogenous mixed solid and cystic appearance [6].

Differentiating benign from malignant lesions may still be difficult on CT [22]. The diagnostic accuracy of CT has been reported to range between $20 \%$ and $90 \%$. This wide range is attributed to the study design, characteristics of the lesions and the presence of lesions with atypical features [7]. Fisher et al. [26] reported accurate specific diagnosis in only $39 \%$ and accurate prediction of malignant potential in $61 \%$ of pancreatic cystic neoplasms using modern CT equipments with expert interpretation.

MRI: The features of pancreatic cystic lesions on MRI are rather similar to CT and accuracy more or less similar to CT has been reported [26].

Endoscopic Ultrasound (EUS): There had been initial enthusiasm for EUS in diagnosing cystic tumours of the pancreas with a wide range of diagnostic accuracy reported ranging from $40 \%$ to $96 \%$. However, EUS has limited ability to differentiate benign from malignant tumours although its main value is to obtain cyst fluid for analysis [4] [5] [11].

Positron Emission Tomography (PET): PET has been advocated for diagnosing and staging cancers. However, PET is unable to differentiate borderline from malignant pancreatic cystic tumours and there is no reliable evidence to validate its accuracy over CT and MRI. Its use, therefore, is limited as a diagnostic tool for pancreatic cystic tumours [11].

Transabdominal ultrasound has its well known limitations in imaging the whole of the pancreas and its sensitivity is limited by the overlying bowel gas [7].

ERCP is the most sensitive modality to detect the main pancreatic duct lesions [1]. In 
some of cases, which has been reported to be up to $50 \%$ of the lesions, the diagnosis of IPMN can be established if a patulous papilla with mucin extrusion is found, a sign called "fish eye" sign [7] [23]. The use of ERCP as the primary diagnostic tool is, however, not recommended [7].

\subsection{Role of Biochemical Markers}

A. Serum markers: Serum carcinoembryonic antigen (CEA) and CA 19 - 9 are not useful in diagnosing cystic tumours of the pancreas as they are in pancreatic adenocarcinoma [6]. Most cystic neoplasms of the pancreas would have normal levels [6]. The sensitivity and specificity are low $(<50 \%)[6]$.

\section{B. Cystic fluid examination:}

1) Image or EUS guided aspiration of the cyst fluid to examine the macroscopic appearance (e.g. aspiration of mucinous fluid in MCN and glycogen rich cells in SCN) is often of little help and so are the amylase level and cytological examination of the cyst fluid [6] [25].

2) Tumour markers in the cyst fluid could, however, be of some help [27].

Carcinoembryonic antigen: (CEA) is regarded as the most useful marker in the cyst fluid to diagnose mucinous lesions [27]. Levels higher than $400 \mathrm{ng} / \mathrm{mL}$ are indicative of mucinous adenocarcinoma or mucinous cystadenoma with a malignant potential [6]. The basic use of CEA is to differentiate mucinous (where CEA is usually but not always high) and serous lesions (where the level is low) [7]. The sensitivity and specificity of CEA in this regard are $50 \%-100 \%$ and $77 \%-95 \%$ respectively [7].

High cyst fluid levels of CA-125 and CA 72-4 are suggestive of a malignant or premalignant potential [6].

Cyst fluid CA 19-9 is of a much limited value in benign and malignant cystic tumours compared with pancreatic adenocarcinoma [6].

C. Genetic markers: The genetic markers such as K-ras mutation have not been well studied in cystic tumours of the pancreas [6]. Furthermore, these genetic tests may not be readily available in the hospital setting.

\section{Management}

Management decision depends on how confident the preoperative diagnosis is which is mainly based on imaging.

As the treatment is essentially surgical because chemotherapy and radiotherapy have no defined role here [10] and provided that pseudocyst has been excluded, there are usually four main questions to answer:

(A) Decision to operate

(B) What operation to choose

(C) Open or laparoscopic

(D) How to manage the spleen

(A) Decision to operate or manage conservatively: The objectives of surgical treatment are to treat symptoms, excise malignant lesions and prevent the progress of a 
potentially malignant lesion into a malignant spreading one.

Cystic serous neoplasms ( $\mathrm{SCN}$ ) can generally be observed unless they are symptomatic which would often be the case in bigger tumours (larger than $4 \mathrm{~cm}$ ) [23]. SCN could increase in size or become symptomatic during the conservative follow up, therefore changing the management from conservative to surgical [8].

Mucinous tumours: The teaching in the past has always been to remove all mucin producing neoplasms [11] and although many of these tumours would still be excised in modern surgical practice, a more tailored selective approach should, however, be adopted.

Mucinous cystic neoplasms (MCN) should be resected in view of their malignant potential particularly that most patients are young with a long life expectancy [11].

Main duct intraductal papillary neoplasms (IPMN) should be resected, unless the tumour is small (less than $10 \mathrm{~mm}$ ) in high risk patients. Nevertheless, all patients regardless of the operative risk should be offered surgery if there is a preoperative evidence of intramural nodule as there is evidence that these tumours have a high risk of invasiveness, not only because of their potential malignant transformation but also because it is difficult to be sure of the nature of the existing lesion preoperatively [8] [17].

Branch duct IPMN tumours can be observed if they are less than $3 \mathrm{~cm}$ in size with no suspicious features on imaging [6]. Small branch duct IPMN that are less than $30 \mathrm{~mm}$ in diameter and do not contain mural nodule on imaging rarely have a malignant potential [11].

There is, however, a school of thought that in absence of risk factors for malignancy and/or worrisome features on radiological assessment, mucin producing cystic lesions of the pancreas (IPMN and MCN) can be conservatively managed as long as the diameter does not exceed $4 \mathrm{~cm}$ provided that the patient is followed up promptly [28]. Understandably, vigilance has to be exercised if the latter policy is followed.

SPPN: Due to their variable behaviour described above and in spite of the general benign nature of solid pseudopapillary neoplasms (SPPN), they should be resected particularly that they often occur in young patients [20].

For all tumours, if it remains impossible to make a definitive diagnosis after all the above described investigations, resection should be considered [22].

Spenilli et al. [29] reported that cystic pancreatic neoplasms occur in $0.7 \%$ of hospital patients, increase in $19 \%$ over 16 months, and are likely (60\%) to be malignant in patients older than 70 years. Therefore, they recommend surgical excision for pancreatic cysts that are increasing under observation, symptomatic, or detected radiologically in fit older patients.

Suggested steps in decision making [11] [13] [27]:

Step 1: Exclusion of pseudocys by CT and/or MRI

Step 2: Once the pseudocyst has been excluded, cystic tumours can be classified into:

(A) Symptomatic tumours which should be resected

(B) Asymptomatic tumours which, again, could be:

(1) Tumours with typical features on imaging: 
SCN: observe unless larger than $4 \mathrm{~cm}$,

MCN and SPPN: should be resected,

IPMN: main duct tumours should be resected whilst branch duct tumours should be managed as explained above.

(2) Tumours with indeterminate features: which in turn could be

(a) Suspicious tumours should be resected.

(b) Simple or compound cysts and lesions with atypical features: individual decisions have to be made e.g. further investigations by EUS, FNA or biochemical markers.

Step 3: For the last category of tumours where definitive diagnosis can not be made with confidence preoperatively, a decision has to be finalised whether to operate or observe in the light of understanding of the behaviour of individual tumours.

(B) Selection of the operation: Depending on the location of the tumours described above, the operations available are: pancreaticoduodenectomy, distal pancreatectomy, total pancreatectomy, segmental resection such as middle pancreatectomy and enucleation [11] [30]. Distal pancreatectomy is the most commonly performed operation [27].

Because of their location, the usual operation for MCN is distal pancreatectomy [11].

The objective of treatment in IPMN is to achieve a surgical negative resection (R0 resection) [11]. That can be achieved by partial pancreatectomy in most cases, depending on the location of the tumours [11]. Because IPMN is characterised by multifocality, total pancreatectomy has been advocated [11] [31] [32]. Advocates of this policy argue on the basis that up to $30 \%$ of patients will have multifocality at the time of diagnosis [11] [33] [34]. Furthermore, achieving clear margins does not necessarily mean there would be no missed foci in the pancreatic tissue left behind in the context of a multifocal disease. However the policy of total pancreatectomy can be criticised as there is no genuine reason for the patients to suffer the disabling endocrine and exocrine sequences of total pancreatectomy for a disease (IPMN) where the recurrence rate in malignant IPMN is similar in total and partial R0 pancreatectomy [11] [35] [36]. Total pancreatectomy has significant metabolic endocrine and exocrine consequences [14]. In brief, in terms of endocrine effects, there would be less need for insulin compared to partial pancreatectomy as there would be no source for glucagon but the therapeutic window is narrow resulting in frequent postprandial hypoglycaemia which initiates a cerebral autonomic response that attenuates epinephrine secretion from the adrenal [14]. This reduction causes episodes of diabetic unawareness which when combined with insulin insensitivity is termed brittle diabetes, a term described in 1930s [14]. The exocrine insufficiency is also significant. Even with aggressive pancreatic enzyme replacement of up to $1,200,000 \mathrm{IU}$ of lipase per meal in combination with proton pump inhibitors to minimize the early inactivation of the enzymes by gastric acid, these patients often have moderate steatorrhoea and glucose malabsorption which further complicates diabetes management [14]. These patients may need up $5000 \mathrm{kcal}$ a day to maintain their body weight [14]. It has been a practice in some centres [14] [17] to attempt partial pancreatectomy according to the location of IPMN and perform intraoperative frozen section to make sure margins are clear. If there is evidence of severe dys- 
plasia or invasive cancer, resection should be extended up to total pancreatectomy although there is some evidence that this may not be necessary for non invasive tumours [37].

SPPN in the pancreatic head should be treated with pancreaticoduodenectomy whilst those in the body and tail can be treated with distal pancreatectomy [18].

Enucleation, which is traditionally the treatment of benign pancreatic endocrine tumours, has been practised for pancreatic cystadenomas (serous and mucinous) [38] and branch duct IPMN [39]. Tumours suitable for enucleation are the benign tumours which should be ascertained intraoperatively, small and superficial tumours where the main pancreatic duct is not expected to be jeopardised by enucleation [38] [40]. The size suitable for enucleation is debateable and it had been estimated to be less than 4 $\mathrm{Cm}$ although some authors would perform enucleation for tumours up to $6 \mathrm{~cm}$ [29] and others would only recommend enucleation for tumours less than $2 \mathrm{~cm} \mathrm{[40]-[42].}$ Advantages are less operating time, less blood loss, quicker recovery and preservation of more pancreatic parenchyma with less chance of endocrine and exocrine consequences compared to resection [38]. There is, however, more chance of developing pancreatic fistula with enucleation [38]. Pancreatic fistula has been defined as draining more than $30 \mathrm{~mL}$ of amylase rich fluid a day [23], persistent draining of lipase rich fluid for 6 days [43] or radiological draining of lipase rich fluid [43]. It is argued that the reason for most papers to report more pancreatic fistula than reality is that most operations were performed for endocrine tumours which are different from cystadenomas in that the latter have fibrous capsules the dissection along which would decrease the incidence of pancreatic fistula [38]. Long follow up after enucleation is lacking [38]. Ge et al. reported no recurrence with enucleation after a follow up period of up to 67 months, which is probably an unnecessary conclusion in a series of benign tumours. Furthermore, no new onset diabetes was reported in the same paper [38].

Central pancreatectomy (middle pancreatectomy, middle segment pancreatectomy) has been proposed as a better alternative to enucleation for benign or low grade tumours including pseudopapillary tumours larger than the size suitable for enucleation located in the central pancreas i.e. in the neck and proximal body [40] [44] [45]. For IPMN, it is most suitable for branch duct tumours [40]. In central pancreatectomy, the proximal stump is oversewn and the distal stump is anastomosed to the jejunum using a Roux-en-Y reconstruction but some authors have described anastomosing the distal stump to the stomach [40] [44] [45]. Frozen sections could be performed and central pancreatectomy should be abandoned if malignancy is found [46]. Advantages over formal pancreaticoduodenectomy and distal pancreatectomy are preservation of pancreatic endocrine and exocrine function, preservation of gastrointestinal continuity and preservation of spleen [40]. Advocates of central pancreatectomy further argue that the effect of pancreatic resection on these functions are unpredictable in patients with apparently normal pancreas because factors such as fibrosis of the pancreatic remnant, Wirsung duct obstruction and pre-existing chronic pancreatitis could affect the functional outcome [44]. However, central pancreatectomy, which leaves two ends of the 
pancreatic duct would, at least in theory, double the chance of pancreatic fistula [40] [46] [47]. Furthermore, the incidence of endocrine and exocrine functional effects after formal pancreatectomy is rare [47]. Moreover, the spleen can often be preserved in more formal resection [47]. Central pancreatectomy is not an oncological operation and what appears to be a benign tumour could turn to be a malignant one postoperatively [47].

(C) Laparoscopic versus open approach: Generally speaking, all the available operations, pancreaticoduodenectomy, distal pancreatectomy, total pancreatectomy, segmental resection such as middle pancreatectomy and enucleation, can, in principle, be performed open or laparoscopically [12] [17] [48]. Benign lesions such as SCN and potentially malignant lesions such as MCN and IPMN are more suitable for laparoscopic approach than the frankly malignant tumours where the chance of open conversion is less in the former [44]. There is less blood loss with laparoscopic resection [17]. Gumbs et al. [17] have reported less operating time and less hospital stay, less incidence of bile leak and gastric volvolus in laparoscopic compared with open pancreatectomy for IPMN although the number of pancreatic fistula was the same in both groups. Laparoscopic pancreatic surgery has been relatively slow to develop compared with other areas of laparoscopic surgery due to technical challenges of this complex surgery, the difficulty in obtaining adequate operative field for the retroperitoneal structures and the anxiety about serious complications [48]. Furthermore, localizing the pancreatic tumours often requires tactile feedback which is largely lost in laparoscopic surgery and that is why many surgeons use laparoscopic ultrasound or laparoscopically assisted techniques [48]. The learning curve is expectedly long and slow. Laparoscopic distal pancreatectomy is more widely practised than pancreaticoduodenectomy [48]. This is because of the perceived difficulty in dissecting the neck of pancreas off the portal vein and the duodenum off the superior mesenteric vessels and performing the complex reconstruction [17]. Moreover, the nature of the lesions in the distal pancreas, being more often benign or low grade neoplasms with less concern about the resection margins, has encouraged more laparoscopic distal pancreatectomy compared with pancreaticoduodenectomy [48]. Enucleation can be performed laparoscopically with different degrees of success [48]. However, the incidence of pancreatic fistula is higher in laparoscopic compared with open enucleation (being $29 \%$ and $16 \%-23 \%$ respectively) and certainly the lack of tactile feeling particularly applies here [38]. If the lesion can not be adequately localised by laparoscopic ultrasound during enucleation, conversion to open would be indicated [48]. Nevertheless, with the increasing experience in both pancreatic and laparoscopic surgery, the learning curve is on a significant increase.

(D) The question of splenectomy: Spleen should be preserved unless splenectomy is oncologically indicated, [14] [49] [50] the pancreatic lesion is adherent to spleen [13] or the lesion is too large to allow preservation of spleen [12]. Splenic preservation-apart from preventing postsplenectomy sepsis-has been reported to delay the onset of diabetes mellitus but that mostly applies to patients with chronic pancreatitis [40]. Splenic vessels should be preserved wherever possible although spleen preservation with divi- 
sion of the splenic vessels depending on the short gastric vessels has been reported [43] [51] as described below (Warshow technique). However, splenic infarcts (40\%) have been reported after Warshaw technique in cases of enlarged spleen with insufficient short gastric vessels [43] [51]. Infarctions may be partial affecting a segment of spleen or total affecting the whole spleen [43]. Warshaw technique can also be complicated with splenic abscesses [43]. It is, therefore, suggested that Warshaw's technique is contraindicated in cases of splenomegaly [50]. This is entirely understandable anatomically as both the left gastroepiploic and short gastric arteries originate from the distal part of the splenic artery or from its terminal branches. If the splenic artery is ligated proximally, that would leave the spleen dependant on some anastomotic vessels supplying the short gastric arteries from elsewhere in the stomach or possibly the oesophagus which may not be sufficient for the spleen to survive.

\section{A Brief Description of the Technique of Laparoscopic Distal Pancreatectomy with and without Splenectomy}

There are much more papers describing laparoscopic distal pancreatectomy compared with those describing laparoscopic pancreaticoduodenectomy as there is more experience with the former and therefore, the technique of laparoscopic distal pancreatectomy will be summarized here.

Distal pancreatectomy is usually performed with the patient in right lateral position (about 60 degrees) [12] but could be performed in supine position [49].

Four or five ports may be used ( 3 ports $12 \mathrm{~mm}$ each and one $15 \mathrm{~mm}$ port) [12] [27] [49] [52]. Four $5 \mathrm{~mm}$ ports have been used [52]. The epigastric port is used for stomach retraction [49]. Retracting the stomach, the lesser sac is entered by dividing the gastrocolic omentum [49]. The approach used is medial to lateral [43] [49] i.e. dissection towards the spleen with preservation of the short gastric vessels. The tail and body of pancreas are dissected off the retroperitoneum along the embryonic plane and separated from the splenic flexure [49]. The splenic artery is dissected off the pancreas using a combination of blunt and bipolar energy dissection and preserved [49]. Once a segment of the artery is freed, it is elevated and held up to facilitate dissection [49]. The pancreas is dissected off the splenic vein using a combination of blunt and bipolar energy dissection [49]. Further dissection of the pancreas off the splenic artery is accomplished with the dissection heading towards the splenic hilum [49]. The tail of pancreas is freed from the splenic hilum [49]. The pancreas, now completely free, is transacted using a vascular stapling device [49]. The pancreatic neck can be divided either by ultrasound dissector or stapler [27] [43] [45] [52]. Stapler is preferred [43]. The stapler used to divide the pancreatic neck should be fired slowly [12]. If splenectomy is performed, the splenic artery is clipped proximally [12]. The splenic artery and vein are divided with the pancreatic neck [43]. If spleen is preserved, splenic vessels are either preserved with ligation of pancreatic branches using clips or ultrasound dissector or resected with the distal pancreas using a stapler making sure the short gastric vessels are preserved [43] [52]. The splenic vessels could be divided twice, firstly at the level of the 
pancreatic neck and then close to the splenic hilum, and if the spleen is going to be preserved, anastomotic branches to the short gastric vessels at the hilum should be preserved. Different techniques have been described to address the pancreatic stump including staplers alone, augmented by fibrin sealant, sutures or omental patch or suture closure if transaction is carried out using ultrasonic shears [43]. The incidence of pancreatic fistula is not related to the technique of dealing with the pancreatic stump [43]. The specimen is put in a plastic bag then a $4 \mathrm{~cm}$ incision is made in the left flank and the specimen is removed [12] [43] [49]. Particular attention is paid to avoid spillage of the tumour [12]. A suction drain is usually needed [43]. Following the same principles, central pancreatectomy could be performed where the proximal stump is secured and the distal stump is used for pancreaticojejunal or pancreaticogastric anastomosis [44]. The expected technical difficulty in performing these anastomosis is quite obvious here.

\section{Follow-Up}

Patients who have resection for benign MCN do not need follow up as these tumours do not recur in the remaining pancreas whilst patients who have resection for a malignant MCN should be followed up with CT as there a chance of local recurrence and haematogenous metastasis [11].

For asymptomatic branch duct IPMN which are observed, follow up is required.

It has been suggested [6] that these patients should have a yearly follow up if the lesions are $<10 \mathrm{~mm}$, a 6 to 12 months follow up if the lesions are $10-20 \mathrm{~mm}$ and a 6 months follow up if the lesions are $>20 \mathrm{~mm}$. If here is an unfavourable progress during the follow up as defined by sequential growth, appearance of new symptoms, appearance of intramural nodules, increase in the cyst size to more than $30 \mathrm{~mm}$ or dilatation of the main pancreatic duct to more than $6 \mathrm{~mm}$, resection would be indicated [6]. If there is no change during the follow up, the interval of follow up can be increased after two years although long term follow up is recommended [11].

There is a real risk of recurrence (up to 70\%) after curative resection for IPMN and that applies to those who have resection for low and high grade dysplasia [11]. Therefore, follow up both clinically and with $\mathrm{CT}$ is essential as some patients would need further resection [10].

\section{Conclusion}

Cystic tumours of the pancreas are relatively rare tumours that are challenging to manage. A general plan of management should be in place when the initial diagnosis is made which should be refined during the subsequent stages of management. There is no unified agreement as to what the best treatment is. However, resection should always be considered if there are features suspicious for malignancy. Laparoscopic surgery has an increasing role to play in this field.

\section{References}

[1] Tanaka, M., Fernández-del Castillo, C., Adsay, V., et al. (2012) International Consensus 
Guidelines 2012 for the Management of IPMN and MCN of the Pancreas. Pancreatology, 12, 183-197. http://dx.doi.org/10.1016/j.pan.2012.04.004

[2] Fritz, S. and Lerch, M.M. (2015) Natural History and Management of Intraductal Papillary Mucinous Neoplasms: Current Evidence. Viszeralmedizin, 31, 25-30. http://dx.doi.org/10.1159/000375186

[3] Zhang, X.P., Yu, Z.X., Zhao, Y.P., et al. (2016) Current Perspectives on Pancreatic Serous Cystic Neoplasms: Diagnosis, Management and Beyond. World Journal of Gastrointestinal Surgery, 8, 202-211. http://dx.doi.org/10.4240/wjgs.v8.i3.202

[4] Kawaguchi, Y. and Mine, T. (2016) Endoscopic Approach to the Diagnosis of Pancreatic Cystic Tumor. World Journal of Gastrointestinal Oncology, 8, 159-164. http://dx.doi.org/10.4251/wigo.v8.i2.159

[5] Chiang, A.L. and Lee, L.S. (2016) Clinical Approach to Incidental Pancreatic Cysts. World Journal of Gastroenterology, 22, 1236-1245. http://dx.doi.org/10.3748/wjg.v22.i3.1236

[6] Ng, D.Z., Goh, B.K., Tham, E.H., et al. (2009) Cystic Neoplasms of the Pancreas: Current Diagnostic Modalities and Management. Annals Academy Medicine Singapore, 38, 251259.

[7] Hutchins, G.F. and Draganov, P.V. (2009) Cystic Neoplasms of the Pancreas: A Diagnostic Challenge. World Journal of Gastroenterology, 15, 48-54.

http://dx.doi.org/10.3748/wjg.15.48

[8] Köksal, A.S., Asil, M., Turhan, N., et al. (2004) Serous Microcystic Adenoma of the Pancreas: Case Report and Review of the Literature. Turkish Journal of Gastroenterology, 15, 183-186.

[9] Duraisami, P.R., Malaichamy, V. and Chithambaram, L. (2015) Serous Microcystadenoma of Pancreas. Journal of Clinical and Diagnostic Research, 9, 9-10.

[10] Farrell, J.J. (2015) Prevalence, Diagnosis and Management of Pancreatic Cystic Neoplasms: Current Status and Future Directions. Gut Liver, 9, 571-589.

http://dx.doi.org/10.5009/gnl15063

[11] Fritz, S., Warshaw, A.L. and Thayer, S.P. (2009) Management of Mucin-Producing Cystic Neoplasms of the Pancreas. Oncologist, 14, 125-136. http://dx.doi.org/10.1634/theoncologist.2008-0200

[12] Mizutani, S., Nakamura, Y., Ogata, M., et al. (2009) A Case of Giant Mucinous Cystic Neoplasm of the Pancreas Resected with Laparoscopic Surgery. Journal of Nippon Medical School, 76, 212-216. http://dx.doi.org/10.1272/jnms.76.212

[13] Brugge, W.R. (2015) Diagnosis and Management of Cystic Lesions of the Pancreas. Journal of Gastrointestinal Oncology, 6, 375-388.

[14] Heidt, D.G., Burant, C. and Simeone, D.M. (2007) Total Pancreatectomy: Indications, Operative Technique, and Postoperative Sequelae. Journal of Gastrointestinal Surgery, 11, 209-216. http://dx.doi.org/10.1007/s11605-006-0025-7

[15] Goh, B.K. (2015) International Guidelines for the Management of Pancreatic Intraductal Papillary Mucinous Neoplasms. World Journal of Gastroenterology, 21, 9833-9837. http://dx.doi.org/10.3748/wjg.v21.i34.9833

[16] Oláh, A. and Kollár, D. (2015) Surgical Aspects of Intraductal Papillary Mucinous Neoplasms of the Pancreas. Chirurgia, 110, 413-417.

[17] Gumbs, A.A., Grès, P., Madureira, F.A. and Gayet, B. (2008) Laparoscopic vs. Open Resection of Noninvasive Intraductal Pancreatic Mucinous Neoplasms. Journal of Gastrointestinal Surgery, 12, 707-712. http://dx.doi.org/10.1007/s11605-007-0311-z

[18] Zeytunlu, M., Firat, O., Nart, D., et al. (2004) Solid and Cystic Papillary Neoplasms of the 
Pancreas: Report of Four Cases. Turkish Journal of Gastroenterology, 15, 178-182.

[19] Patil, T.B., Shrikhande, S.V., Kanhere, H.A., Saoji, R.R., Ramadwar, M.R. and Shukla, P.J. (2006) Solid Pseudopapillary Neoplasm of the Pancreas: A Single Institution Experience of 14 Cases. HPB, 8, 148-150. http://dx.doi.org/10.1080/13651820510035721

[20] Ozguven, B.Y., Tuncel, D., Pola,t N., et al. (2015) Solid-Pseudopapillary Neoplasm of the Pancreas: Clinicopathologic and Immunohistochemical Analysis of Nine Cases. Indian Journal of Pathology \& Microbiology, 58, 292-295. http://dx.doi.org/10.4103/0377-4929.162833

[21] Kadhirvel, V., Ramu, S., Mishra, N., Adaikalam, M.L.S. and Venkatesan, R. (2015) Serous Microcystic Adenocarcinoma of Pancreas Infiltrating into Spleen: A Case Report. Journal of Clinical and Diagnostic Research, 9, ED01-ED02. http://dx.doi.org/10.7860/jcdr/2015/12869.6439

[22] Visser, B.C., Muthusamy, V.R., Yeh, B.M., Coakley, F.V. and Way, L.W. (2008) Diagnostic Evaluation of Cystic Pancreatic Lesions. HPB, 10, 63-69.

http://dx.doi.org/10.1080/13651820701883155

[23] Sand, J. and Nordback, I. (2005) The Differentiation between Pancreatic Neoplastic Cysts and Pancreatic Pseudocyst. Scandinavian Journal of Surgery, 94, 161-164.

[24] Fischer, C.P., Pope, I. and Garden, O.J. (2001) Mucinous Cystic Tumour of the Pancreas Presenting with Acute Pancreatitis. $H P B, 3,271-273$. http://dx.doi.org/10.1080/136518201753335782

[25] Stade, M., Sasson, A.R., Oleynikov, D., Stothert, J. and Thompson, J. (2005) Influence of Pre-Operative Diagnosis and Frozen Section on Operative Management of Pancreatic Cystic Lesions. HPB, 7, 235-237. http://dx.doi.org/10.1080/13651820510037648

[26] Fisher, W.E., Hodges, S.E., Yagnik, V., et al. (2008) Accuracy of CT in Predicting Malignant Potential of Cystic Pancreatic Neoplasms. HPB, 10, 483-490. http://dx.doi.org/10.1080/13651820802291225

[27] Kwiatkowski, A.P., Kowalewski, P.K. and Paśnik, K. (2015) Laparoscopic Approach to Distal Pancreatectomy in Pancreatic Cystic Neoplasms-Report of Three Cases and Literature Review. Wideochir Inne Tech Maloinwazyjne, 10, 499-503. http://dx.doi.org/10.5114/wiitm.2015.54058

[28] Del Chiaro, M. and Verbeke, C. (2015) Cystic Tumors of the Pancreas: Opportunities and Risks. World Journal of Gastrointestinal Pathophysiology, 6, 29-32. http://dx.doi.org/10.4291/wjgp.v6.i2.29

[29] Spinelli, K.S., Fromwiller, T.E., Daniel, R.A., et al. (2004) Cystic Pancreatic Neoplasms: Observe or Operate. Annals of Surgery, 239, 651-657. http://dx.doi.org/10.1097/01.sla.0000124299.57430.ce

[30] Glanemann, M., Shi, B., Liang, F., et al. (2008) Surgical Strategies for Treatment of Malignant Pancreatic Tumors: Extended, Standard or Local Surgery? World Journal of Surgical Oncology, 6, 123. http://dx.doi.org/10.1186/1477-7819-6-123

[31] Bendix Holme, J., Jacobsen, N., Rokkjaer, M. and Kruse, A. (2001) Total Pancreatectomy in Six Patients with Intraductal Papillary Mucinous Tumour of the Pancreas: The Treatment of Choice. HPB, 3, 257-262. http://dx.doi.org/10.1080/136518201753335539

[32] Chari, S.T., Yadav, D., Smyrk, T.C., et al. (2002) Study of Recurrence after Surgical Resection of Intraductal Papillary Mucinous Neoplasm of the Pancreas. Gastroenterology, 123, 1500-1507. http://dx.doi.org/10.1053/gast.2002.36552

[33] Kaneko, T., Nakao, A., Inoue, S., et al. (2001) Intraoperative Ultrasonography by HighResolution Annular Array Transducer for Intraductal Papillary Mucinous Tumors of the 
Pancreas. Surgery, 129, 55-65. http://dx.doi.org/10.1067/msy.2001.109118

[34] Kaneko, T., Nakao, A., Nomoto, S., et al. (1998) Intraoperative Pancreatoscopy with the Ultrathin Pancreatoscope for Mucin-Producing Tumors of the Pancreas. Archives of Surgery, 133, 263-267. http://dx.doi.org/10.1001/archsurg.133.3.263

[35] Jang, J.Y., Kim, S.W., Ahn, Y.J., et al. (2005) Multicenter Analysis of Clinicopathologic Features of Intraductal Papillary Mucinous Tumor of the Pancreas: Is It Possible to Predict the Malignancy before Surgery? Annals of Surgical Oncology, 12, 124-132. http://dx.doi.org/10.1245/ASO.2005.02.030

[36] Maire, F., Hammel, P., Terris, B., et al. (2002) Prognosis of Malignant Intraductal Papillary Mucinous Tumours of the Pancreas after Surgical Resection. Comparison with Pancreatic Ductal Adenocarcinoma. Gut, 51, 717-722. http://dx.doi.org/10.1136/gut.51.5.717

[37] Pelaez-Luna, M., Chari, S.T., Smyrk, T.C., et al. (2007) Do Consensus Indications for Resection in Branch Duct Intraductal Papillary Mucinous Neoplasm Predict Malignancy? A Study of 147 Patients. American Journal of Gastroenterology, 102, 1759-1764. http://dx.doi.org/10.1111/j.1572-0241.2007.01224.x

[38] Ge, C., Luo, X., Chen, X. and Guo, K. (2010) Enucleation of Pancreatic Cystadenomas. Journal of Gastrointestinal Surgery, 14, 141-147. http://dx.doi.org/10.1007/s11605-009-1023-3

[39] Madura, J.A., Yum, M.N., Lehman, G.A., et al. (2004) Mucin Secreting Cystic Lesions of the Pancreas: Treatment by Enucleation. The American Surgeon, 70, 106-112.

[40] Lavu, H., Knuth, J.L., Baker, M.S., et al. (2008) Middle Segment Pancreatectomy Can Be Safely Incorporated into a Pancreatic Surgeon's Clinical Practice. HPB, 10, 491-497. http://dx.doi.org/10.1080/13651820802356580

[41] Roggin, K.K., Rudloff, U., Blumgart, L.H. and Brennan, M.F. (2006) Central Pancreatectomy Revisited. Journal of Gastrointestinal Surgery, 10, 804-812. http://dx.doi.org/10.1016/j.gassur.2005.11.012

[42] Kiely, J.M., Nakeeb, A., Komorowski, R.A., Wilson, S.D. and Pitt, H.A. (2003) Cystic Pancreatic Neoplasms: Enucleate or Resect? Journal of Gastrointestinal Surgery, 7, 890-897. http://dx.doi.org/10.1007/s11605-003-0035-7

[43] Taylor, C., O’Rourke, N., Nathanson, L., et al. (2008) Laparoscopic Distal Pancreatectomy: The Brisbane Experience of Forty-Six Cases. $H P B, 10,38-42$. http://dx.doi.org/10.1080/13651820701802312

[44] Bassi, C. (2007) Middle Segment Pancreatectomy: A Useful Tool in the Management of Pancreatic Neoplasms. Journal of Gastrointestinal Surgery, 11, 726-729. http://dx.doi.org/10.1007/s11605-007-0179-y

[45] Zhang, R.C., Xu, X.W., Zhou, Y.C., et al. (2014) A Rare Case of Mixed Mucinous Cystadenoma with Serous Cystadenoma of the Pancreas Treated by Laparoscopic Central Pancreatectomy. World Journal of Surgical Oncology, 12, 318. http://dx.doi.org/10.1186/1477-7819-12-318

[46] Brown, K.M., Shoup, M., Abodeely, A., Hodul, P., Brems, J.J. and Aranha, G.V. (2006) Central Pancreatectomy for Benign Pancreatic Lesions. HPB, 8, 142-147. http://dx.doi.org/10.1080/13651820510037611

[47] Reber, H.A. (2007) Middle Pancreatectomy: Why I Rarely Do It. Journal of Gastrointestinal Surgery, 11, 730-732. http://dx.doi.org/10.1007/s11605-007-0188-x

[48] Al-Taan, O.S., Stephenson, J.A., Briggs, C., Pollard, C., Metcalfe, M.S. and Dennison, A.R. (2010) Laparoscopic Pancreatic Surgery: A Review of Present Results and Future Prospects. $H P B, 12,239-243$. http://dx.doi.org/10.1111/j.1477-2574.2010.00168.x 
[49] Boutros, C., Espat, N.J. and Somasundar, P. (2010) Completely Laparoscopic Subtotal Pancreatectomy with Splenic Artery Preservation. Journal of Gastrointestinal Surgery, 14, 171 174. http://dx.doi.org/10.1007/s11605-009-0995-3

[50] Fernández-Cruz, L., Orduña, D., Cesar-Borges, G. and López-Boado, M.A. (2005) Distal Pancreatectomy: En-Bloc Splenectomy vs Spleen-Preserving Pancreatectomy. HPB, 7, 9398. http://dx.doi.org/10.1080/13651820510028972

[51] Fernández-Cruz, L., Martínez, I., Gilabert, R., Cesar-Borges, G., Astudillo, E. and Navarro, S. (2004) Laparoscopic Distal Pancreatectomy Combined with Preservation of the Spleen for Cystic Neoplasms of the Pancreas. Journal of Gastrointestinal Surgery, 8, 493-501. http://dx.doi.org/10.1016/j.gassur.2003.11.014

[52] Fernández-Cruz, L. (2006) Distal Pancreatic Resection: Technical Differences between Open and Laparoscopic Approaches. $H P B, 8,49-56$.

http://dx.doi.org/10.1080/13651820500468059

Submit or recommend next manuscript to SCIRP and we will provide best service for you:

Accepting pre-submission inquiries through Email, Facebook, LinkedIn, Twitter, etc. A wide selection of journals (inclusive of 9 subjects, more than 200 journals)

Providing 24-hour high-quality service

User-friendly online submission system

Fair and swift peer-review system

Efficient typesetting and proofreading procedure

Display of the result of downloads and visits, as well as the number of cited articles

Maximum dissemination of your research work

Submit your manuscript at: http://papersubmission.scirp.org/

Orcontact jct@scirp.org 